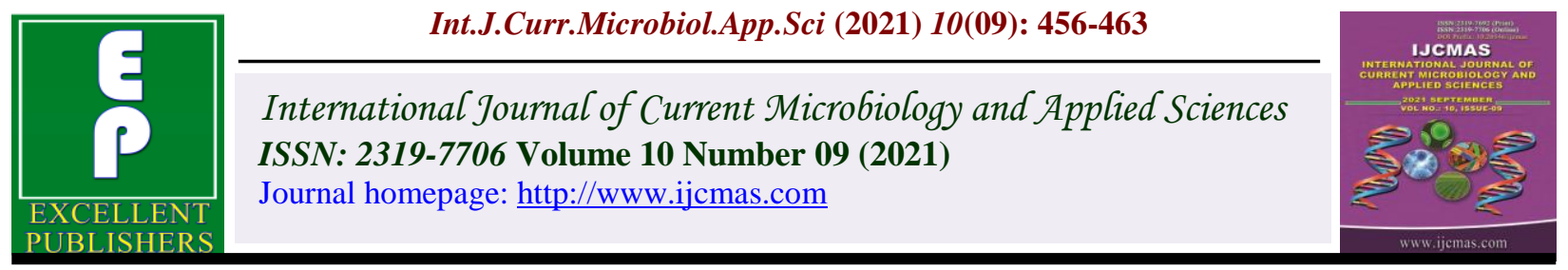

\title{
Evaluation of Antimicrobial Susceptibility Pattern of Pathogenic Bacteria Causing Urinary Tract Infection in a Teaching Hospital
}

\author{
Pradha Velu ${ }^{1}$, Ramya Rengaraj ${ }^{2}$, Hemalatha Gurumurthy ${ }^{2 *}$ and Saleem Mohamed Ali ${ }^{2}$ \\ ${ }^{1}$ Department of Microbiology, Sri Lakshminarayana Institute of Medical Sciences, \\ Puducherry-605502, India \\ ${ }^{2}$ Department of Microbiology, Melmaruvathur Adhiparasakthi Institute of Medical Sciences \\ and Research, Tamil Nadu-603319, India \\ *Corresponding author
}

Keywords

Urinary tract infection, uropathogen. antibiotic susceptibility pattern, ESBL, multi drug resistance

Article Info

Accepted: 15 August 2021 Available Online: 10 September 2021

\section{A B S T R A C T}

To determine the prevalence of pathogenic bacteria causing urinary tract infections and their antibiotics susceptibility pattern from patients reporting in a tertiary care teaching hospital. Study design: Retrospective study. Place and duration of the study: Melmaruvathur Adhiparasakthi Institute of Medical Sciences and Research, January 2017 to December 2019. 7825 Mid-stream urine (MSU) samples collected for culture and sensitivity testing, sent to the laboratory during the period of study were included in this study. The samples were inoculated onto blood agar and MacConkey agar, incubated at $37^{\circ} \mathrm{C}$ for 24 hours. Identification of organisms by biochemical tests and antibiotic susceptibility testing were done by standard microbiological methods. Out of 7825 samples, 3832 showed significant bacteriuria, of which 50\% (1924/3832) were females. Escherichia coli was the most common pathogen isolated, which accounts for $41 \%$ followed by Klebsiella spp. (27\%), Staphylococcus aureus (7\%), Proteus spp. (7\%), Acinetobacter spp. (5\%), Pseudomonas spp. (4\%), Enterobacter spp. (4\%), Citrobacter spp.(4\%), and Serratia spp. (0.9\%). The antimicrobial susceptibility testing of Staphylococcus aureus showed 33\% were Methicillin Resistant Staphylococcus aureus. The antimicrobial susceptibility pattern of gram negative bacilli showed high resistance to cephalosporins followed by carbapenems. Among gram negative bacilli, Klebsiella spp. showed $36 \%$ extended spectrum beta lactamases (ESBL) production. This study determines the trends in antimicrobial sensitivity patterns of uropathogens. It is helpful in the formulation of local antibiotic policy for the hospital and assist clinicians in the rational choice of antibiotic therapy to prevent misuse or overuse of antibiotics so as to prevent emergence of multi drug resistant pathogens. 


\section{Introduction}

Urinary Tract Infection (UTI) is a general term that refers to an infection or inflammation, of any part of the urinary tract caused by microbes such as bacteria, viruses, fungi or parasites. UTI caused by bacteria is one of the most common infections encountered by clinicians worldwide. ${ }^{(1)}$ It is a significant public health problem if not treated early, the complications may renal scaring, end stage renal failure, hypertension finally leading to morbidity and mortality. ${ }^{(2)}$

UTI is classified into two major categories, namely complicated and uncomplicated infections. UTI is complicated when an individual's urinary tract is obstructed or abnormal. In case of uncomplicated UTI, the urinary tract is normal and unobstructed. In uncomplicated UTI, $80 \%-90 \%$ infection is caused by Escherichia coli. ${ }^{(2)}$ Other pathogens include Klebsiella spp., Pseudomonas spp. and other non-fermenters, Enterococcus spp., Staphylococcus spp., Enterobacter spp., Citrobacter spp., and Proteusspp.. As compared to non-pathogenic bacteria, the bacteria which are responsible for causing UTI have more aggressive virulence factors which enhance their host cell attachment, colonization as well as invasion abilities. These bacteria evade the immune system of the host by the help of certain virulence factors that comprises of various cellular components such as pili, capsule, lipopolysaccharides, and various other cell surface structures. ${ }^{(3)}$

Certain human anatomical as well as physiological factors are also responsible for increasing the incidence of UTI, for example, length of the urethra is shorter in females as compared to males which increases the chance of acquiring UTI. Similarly, incomplete emptying of bladder particularly in old age results in accumulation of residual urine in the bladder and vesicoureteral reflux that frequently occurs among pregnant women can predispose host to UTI. Infants, pregnant women, patients with spinal cord injuries, diabetes, multiple sclerosis, acquired immunodeficiency disease syndrome or underlying urologic abnormalities are subjects at increased risk for UTI. In addition, catheterassociated UTI is the most common nosocomial infection. ${ }^{(4)}$

Worldwide, about 150 million people are diagnosed with UTI each year; nearly $10 \%$ of people will experience UTI at least once in their life-time ${ }^{(5)}$ Women are more susceptible to UTI than men. Over $50 \%$ of all women will experience at least one UTI during their lifetime, with $20-30 \%$ experiencing recurrent UTI. ${ }^{(2)}$ Recurrent UTIs warrant the use of multiple courses of antibiotic therapy. Since last decade there is alarming changes in drug susceptibility pattern in bacterial agents causing UTI. Antibiotic resistance is becoming a major concern in treating complicated and uncomplicated UTI.

Broad-spectrum antibiotics are often prescribed to treat UTI and treatment is started empirically without ordering culture and sensitivity testing. Since, studies concerning culture and sensitivity profiles of uropathogens have been performed over decades, there have been drastic changes in the sensitivity profiles over time and more so due to indiscriminate as well illogical use of antibiotics. ${ }^{(6)}$ This inappropriate and nonjudicious usage of antibiotics has resulted in the development of antibiotic resistance in bacteria, worldwide; leading to the emergence of multi-resistant strains of bacterial pathogens. Therefore, choice of suitable antibiotics is a major determinant of appropriate therapy and prevention of chronic complications. There is change in microbial distribution and resistance pattern from place to place which makes it mandatory to monitor 
organisms distribution and their resistance pattern in a particular area. Hence, we endeavoured to study the current trend of causative organisms and their antibiotic susceptibility pattern in patients with UTI.

\section{Materials and Methods}

\section{Study design}

A retrospective study conducted in the department of Microbiology over a three-year period from January 2017-December 2019. Urine samples collected from clinically suspected cases of UTI in both hospitalized and out patients, irrespective of their age and sex is included in the study.

\section{Sampling method}

Most of the samples are clean catch midstream samples and the rest, catheter samples and supra pubic aspirates.

\section{Sample processing}

All the samples are processed in laboratory within two hours of collection. Microscopic examination for pus cells, RBCs, casts, epithelial cells, microbes and culture on MacConkey agar and 5\% sheep blood agar for bacterial growth were done. Significance of the isolates were assessed based on microscopic findings as well as the clinical features by Kass's criteria. ${ }^{(7)}$ Colony counts $<10^{5}$ also are considered significant on an individual basis by correlating the microscopic findings, type of specimen and the clinical history. Identification of the isolates were done by standard bacteriological methods. ${ }^{(8)}$

Antibiotic susceptibility testing of the isolates were performed by Kirby- Bauer disc diffusion method on Mueller Hinton Agar as per Clinical Laboratory Standard Institute guidelines. ${ }^{(9)}$ using Hi-Media, India antibiotic discs. Antibiotic discs tested were amoxyclav (30 $\mu \mathrm{g} / 10 \mu \mathrm{g}), \quad$ norfloxacin $(10 \mu \mathrm{g})$, ciprofloxacin $(5 \mu \mathrm{g})$, nitrofurantoin $(300 \mu \mathrm{g})$, co-trimoxazole $(1.25 / 23.75 \mu \mathrm{g})$, cefuroxime $(30 \mu \mathrm{g})$, cefotaxime $(30 \mu \mathrm{g})$, ceftazidime $(30 \mu \mathrm{g})$, gentamicin $(10 \mu \mathrm{g})$, amikacin $(30 \mu \mathrm{g})$, piperacillin-tazobactam $(100 / 10 \mu \mathrm{g})$, imipenem $(10 \mu \mathrm{g})$, cefoxitin $(30 \mu \mathrm{g})$. Using CLSI phenotypic confirmatory tests ${ }^{(8)}$, methicillin resistant Staphylococcus aureus (MRSA) detection by cefoxitin disc, Extended Spectrum Beta Lactamase (ESBL) detection by double disc synergy test (DDST) among the members of Enterobacteriaceae were done.

\section{Quality control}

Quality control was performed using standard strains, Escherichia coli ATCC 25922, Pseudomonas aeruginosa ATCC 27853, and Staphylococcus aureus ATCC 25923.

Considering the differences in the susceptibility pattern of bacterial agents of UTI among community acquired and hospital acquired cases, characterization of the isolates and their antibiotic resistance pattern based on the admission status of the patients was also considered.

\section{Results and Discussion}

\section{Sample distribution}

A total of 7825 urine samples were recruited for the study. Of those 7825, 2683 and 5142 were inpatients (IP) and outpatients (OP) respectively (Table 1). Significant bacterial growth was found in 3832 isolates (Table 2).

\section{Uropathogens isolated}

In significant bacterial growth, the organisms grown in urine samples are Staphylococcus aureus (7\%), Escherichia coli (41\%), Klebsiella (27\%), Proteus (7\%), Acinetobacter 
(5\%), Pseudomonas (4\%), Enterobacter (4\%), Citrobacter (4\%) and Serratia (0.9\%). In this Escherichia coli (896) were most commonly isolated and followed by Klebsiella spp. (624) and the most of the isolates are from Outpatients sample (Chart 1).

\section{Antimicrobial resistance pattern}

Overall 255 isolates of Staphylococcus aureus were obtained from urine samples. The antimicrobial susceptibility testing showed $33 \%$ of these isolates was Methicillin Resistant Staphylococcus aureus. $60 \%$ of Staphylococcus aureus were resistant to nitrofurantoin, followed by gentamicin $26 \%$ and norfloxacin 25\%. Most of the isolates was sensitive to erythromycin and amikacin 95\% and $90 \%$ respectively. The antimicrobial resistance pattern of gram negative bacilli are presented in the table 4. Majority of Gram negative isolates were resistant to cephalosporins followed by carbapenems. Among the Gram negative isolates Klebsiella spp.showed $36 \%$ ESBL production as shown in chart 2.

\section{Prevalence of UTI-gender based}

Urinary tract infection (UTI) is one of the most frequent causes of nosocomial infections. UTI is caused by both Gram-negative and Gram-positive bacteria. The results of present study shows that significant bacteriuria is equally prevalent in both females and males.

The study conducted by Prakash et al., ${ }^{(10)}$ found that UTI with significant bacteriuria is more common among females $(73.57 \%)$ rather than males $(35.14 \%)$. Whereas in contrast to that study a report was presented by Kumar Gauvrav et al., ${ }^{(11)}$ and they showed $52.5 \%$ of male and $47 \%$ of female had significant bacteriuria. This variation may be due to population selection like inclusion of geriatric age group. Young age females are more prone to UTI whereas in older age males gets UTI because of physiological variation.

\section{Uropathogens}

The etiological agents and their susceptibility pattern of UTI may vary according to regions and geographical locations. Knowledge of bacterial aetiology and susceptibility pattern is required for initiating optimal empirical therapy for UTI. In our study the most commonly encountered bacteria are Gram negative bacilli in which Escherichia coli is the most common followed by Klebsiella spp., Proteus spp., Staphylococcus spp., Pseudomonas spp., Acinetobacter spp., Enterobacter spp. and Serratia spp.

This coincides with many of the studies. Study for monitoring antimicrobial resistance across India was conducted by Balaji Veeraraghavan et al., ${ }^{(12)}$ showed that Escherichia coli is the most common isolates over the country. Mohan Kumar et al., ${ }^{(13)}$ reported $48.9 \%$ of $E$. coli causing UTI in a study from Kerala. Klebsiella spp (27\%) isolated in our study which is next highest number to Escherichia coli. Bashir A Fomda et al., ${ }^{(14)}$ in 2018 reported $11.4 \%$ of Klebsiella in Manipur. In our study Staphylococcus aureus (7\%) was isolated which is proportion to the study conducted by Monika Yadav et al., ${ }^{(15)}$

\section{Antimicrobial resistance pattern}

Effective treatment of UTI patients mainly relies on identification of pathogen and antimicrobial susceptibility of pathogens. Monitoring of antimicrobial susceptibility can aid clinicians for prescribing appropriate antibiotics and in prevention of development of drug resistance through commitment to antibiotic stewardship. The present study showed $60 \%$ of Gram negative bacilli are resistant to cefuroxime followed by $45 \%$ to cefotaxime, $30 \%$ to imipenem and $25 \%$ to amikacin. 
Table.1 Distribution of samples

\begin{tabular}{|c|c|c|}
\hline Total & Inpatient & Outpatient \\
\hline $\mathbf{7 8 2 5}$ & 2683 & 5142 \\
\hline
\end{tabular}

Table.2 Significant bacteriuria

\begin{tabular}{|c|c|c|}
\hline Significant growth & Inpatient & Outpatient \\
\hline $\mathbf{3 8 3 2}$ & 1780 & 2052 \\
\hline
\end{tabular}

Out of 3832 samples with significant growth, 1924 (50\%) were female patients. The age of the subjects varied from 18-85 (Table 3).

Table.3 Age distribution of Female patients

\begin{tabular}{|c|c|c|}
\hline Age-interval (years) & No. & Percentage \\
\hline $\mathbf{1 6 - 2 5}$ & 280 & 14.5 \\
\hline $\mathbf{2 6 - 3 5}$ & 422 & 22 \\
\hline $\mathbf{3 6 - 4 5}$ & 385 & 20 \\
\hline $\mathbf{4 6 - 5 5}$ & 398 & 20.5 \\
\hline $\mathbf{5 6 - 6 5}$ & 205 & 11 \\
\hline $\mathbf{6 6 - 7 5}$ & 202 & 10 \\
\hline $\mathbf{7 6 - 8 5}$ & 32 & 2 \\
\hline Total $(\mathbf{N})$ & $\mathbf{1 9 2 4}$ & $\mathbf{1 0 0}$ \\
\hline
\end{tabular}

Table.4 Antibiotic susceptibility pattern: Resistance

\begin{tabular}{|c|c|c|c|c|c|c|}
\hline & Staph aureus & E.coli & $\begin{array}{l}\text { Klebsiella } \\
\text { spp. }\end{array}$ & $\begin{array}{l}\text { Proteus } \\
\text { spp. }\end{array}$ & $\begin{array}{c}\text { Acinetobacter } \\
\text { spp. }\end{array}$ & $\begin{array}{l}\text { Pseudomonas } \\
\text { spp. }\end{array}$ \\
\hline & $\mathrm{N}(\%)$ & $\mathrm{N}(\%)$ & $\mathrm{N}(\%)$ & $\mathrm{N}(\%)$ & $\mathrm{N}(\%)$ & $\mathrm{N}(\%)$ \\
\hline Amoxyclav & $184(72)$ & $873(55)$ & $639(62)$ & $110(40)$ & $67(35)$ & 0 \\
\hline Cefoxitin & $84(33)$ & 0 & 0 & 0 & 0 & 0 \\
\hline Nitrofurantoin & $153(60)$ & $555(35)$ & $330(32)$ & $113(41)$ & $67(35)$ & $85(56)$ \\
\hline Ciprofloxacin & $31(12)$ & $317(20)$ & $289(28)$ & $72(26)$ & $81(42)$ & $38(25)$ \\
\hline Norfloxacin & $64(25)$ & $397(25)$ & $227(22)$ & $83(30)$ & $48(25)$ & $38(25)$ \\
\hline Cefuroxime & $128(50)$ & $825(52)$ & $807(62)$ & 151(55) & $119(62)$ & 0 \\
\hline Cefotaxime & $128(50)$ & $635(40)$ & $467(45)$ & $143(52)$ & 0 & 0 \\
\hline Ceftazidime & $77(30)$ & 0 & 0 & 0 & $125(65)$ & $60(40)$ \\
\hline Amikacin & $26(10)$ & $318(20)$ & $256(25)$ & $83(30)$ & $50(26)$ & $53(35)$ \\
\hline Gentamicin & $66(26)$ & $476(30)$ & $256(25)$ & $94(34)$ & $50(26)$ & $33(22)$ \\
\hline $\begin{array}{l}\text { Piperacillin/ } \\
\text { Tazobactum }\end{array}$ & $64(25)$ & $318(20)$ & $268(26)$ & $88(32)$ & $29(15)$ & $18(12)$ \\
\hline $\begin{array}{c}\text { Cefotaxime/Clav acid } \\
\text { Ceftaz/clav acid }\end{array}$ & $90(35)$ & 397(25) & $467(45)$ & $83(30)$ & $58(30)$ & $53(35)$ \\
\hline Imipenem & 0 & $603(38)$ & $330(32)$ & $55(20)$ & $81(42)$ & $30(20)$ \\
\hline Cotrimoxazole & $179(70)$ & $333(21)$ & $227(22)$ & $132(48)$ & $67(35)$ & 0 \\
\hline Erythromycin & $13(5)$ & 0 & 0 & 0 & 0 & 0 \\
\hline
\end{tabular}




\section{Chart.1 Distribution of Organisms}

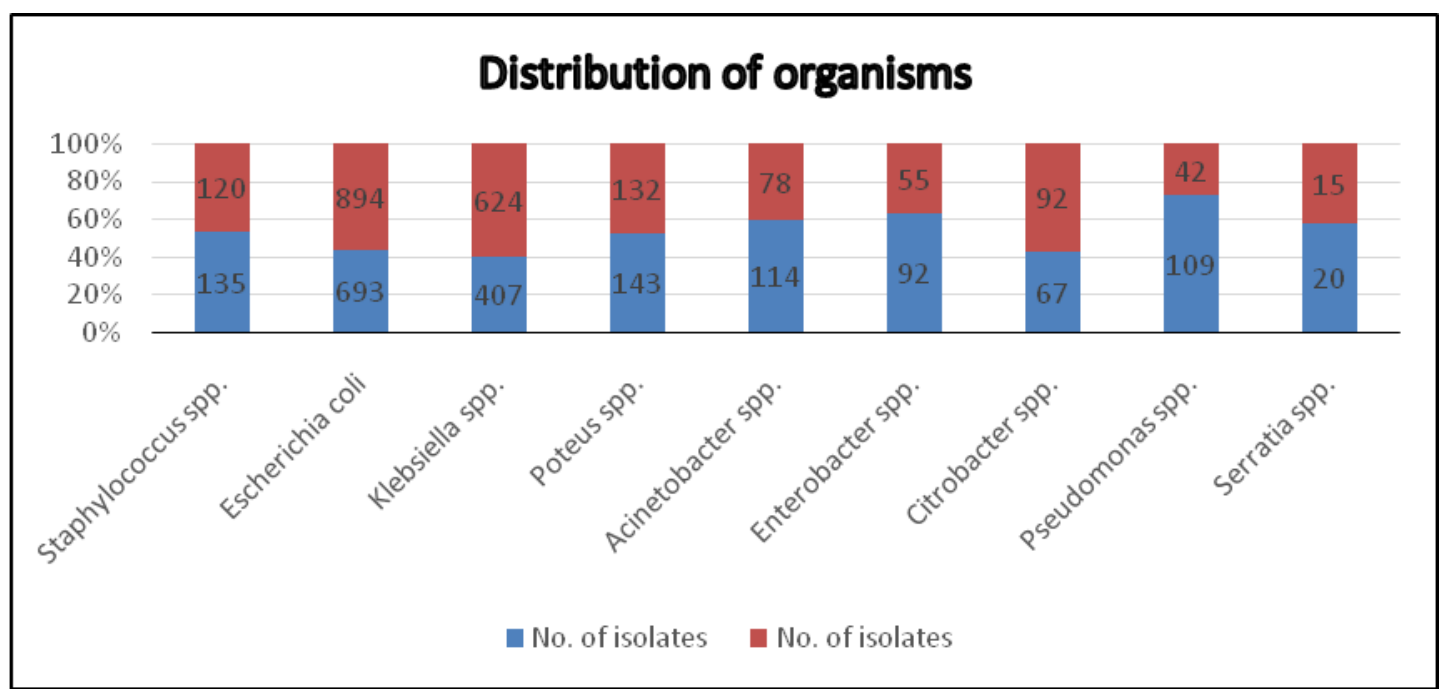

Chart.2 Distribution of MRSA \& ESBL

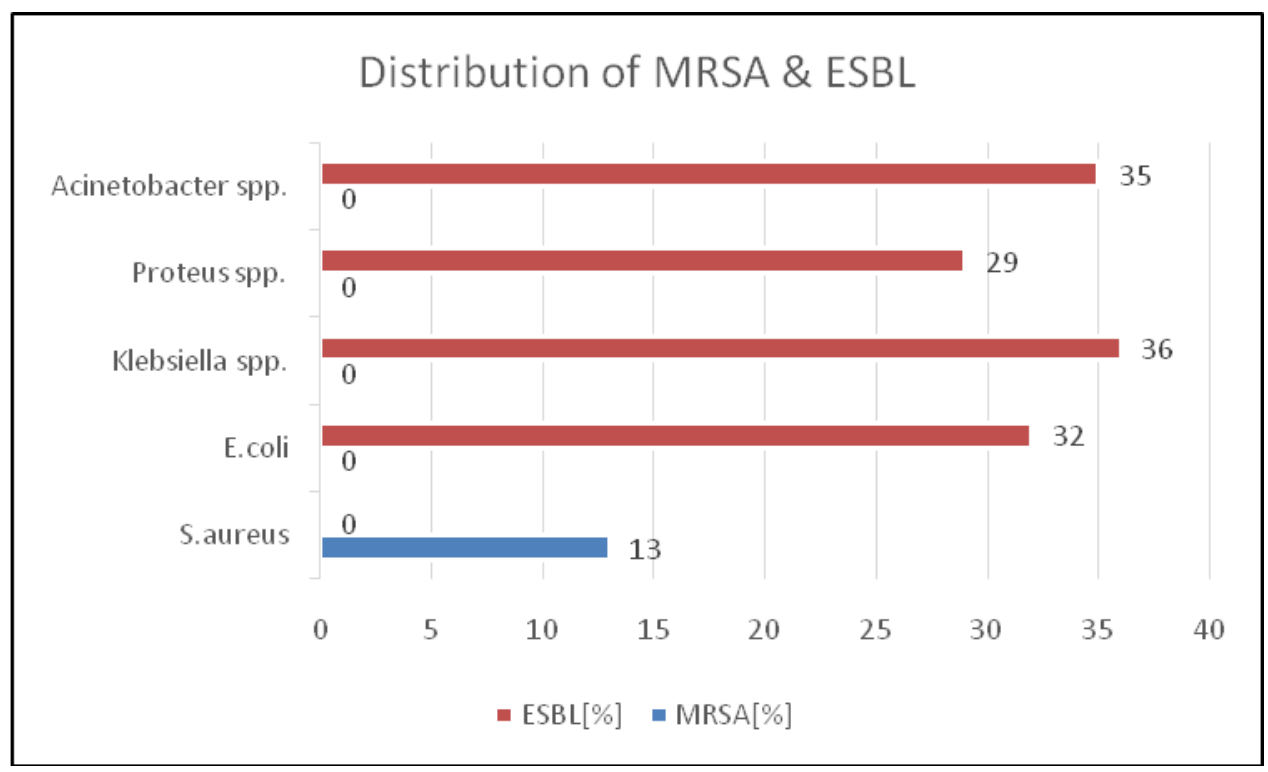

Adane Bitew et al., ${ }^{(4)}$ reported that the ampicillin had the highest resistance $78.4 \%$ followed by trimethoprim/sulfamethoxazole $66.3 \%$ and tetracycline $66.3 \%$ but these drugs we are not routinely using for treatment of UTI.

In the present study has reported Gram negative organisms are more resistant to cephalosporin group of drugs than carbapenems. A similar study conducted by Balaji Veeraraghavan et al., ${ }^{(12)}$ showed carbapenems were the most active, followed by amikacin and piperacillin/tazobactam on gram negative organisms. Among cephalosporins, the activity of cefoxitinwas found superior than the other agents. Moderate activity was noted for fluoroquinolones and aztreonam. In our study most of the isolates are sensitive to norfloxacin and nitrofurantoin. 
As the antibiotic resistance may vary according to region and geographical location, E. coli and Klebsiella spp. are found to be more sensitive to nitrofurantoin, gentamicin, and norfloxacin, while less sensitive to ceftazidime, cefotaxime and carbapenems which is similar to study done by Niranjan et al., ${ }^{(16)}$ in Puducherry, India.

A low degree of resistance to aminoglycoside and fluoroquinolones was observed for both gram negative and gram positive organisms and hence may be helpful in combating severe infections.

The oral antibiotic nitrofurantoin was found to be moderately effective in treatment of UTI in our study. Khameneh et al., ${ }^{(17)}$ and few other Indian studies, also have proved nitrofurantoin as an appropriate agent for first line treatment of community acquired UTI.

\section{Multidrug resistance pattern}

Antimicrobial resistance to multiple drugs is a major clinical problem in treating infections caused by different bacterial pathogens. In our study, 33\% of Pseudomonas spp are multidrug resistant which is followed by $30 \%$ of Escherichia coli and 29\% of Klebsiella spp. The present study show $36 \%$ of Klebsiella produce Extended Spectrum Beta Lactamases. Resistance to imipenem, which is used as last resort drugs in the health care settings was found to be around $20 \%-35 \%$, in our study which is quite alarming.

The high rate of resistance are seen in most commonly prescribing antibiotics, which are easily available in the community, very cheap in terms of cost and hence subject to misuse. It could also be due to the use of antibiotics for other nonhuman purposes such as in livestock rearing and animal husbandry activities, which may contribute to the growing rate of resistance.

\section{Significance of this study}

The strength of this retrospective study is that it evaluated large number of urine samples with significant bacteriuria and highlights the emergence of antimicrobial resistance that provides precise scientific data for appropriate treatment, prevention, and control of UTI. However, the study was a single hospitalbased study and may not represent multiple clinical settings.

This study warrants the observation of multi drug resistant bacterial pathogens causing UTI and its epidemiological survey in healthcare institution. High level of drug resistance against the commonly prescribed drugs necessitates a search for other options. The findings of this study emphasized the need for constant monitoring of specific pathogen and its antimicrobial susceptibility testing. We hope that this study would help the clinicians in deciding the appropriate antibiotic therapy for UTIs and also would help in formulating an antibiotic policy in the hospitals.

\section{Ethical approval}

All authors hereby declare that the research proposal has been examined and approved by the appropriate institutional research and ethics committee (mapims/iec/52/2019) and have therefore been performed in accordance with the ethical standards laid down in the 1964 declaration of helsinki.

\section{References}

1. H. I. Magale, I. A. Kassim, S. A. Odera, M. J. Omolo, W. G. Jaoko, P. E. Jolly. Antibiotic Susceptibility of Organisms Causing Urinary Tract Infection In Patients Presenting At Kenyatta National Hospital, Nairobi. East African Medical Journal. 2015;92(7):333-37.

2. Ayelign B, Abebe B, Shibeshi A, et al., Bacterial isolates and their antimicrobial susceptibility patterns among pediatric patients with urinary tract infections. Turkish Journal of Urology. 2018 Jan;44(1):62-69. DOI: 
10.5152/tud.2017.33678. PMID: 29484230; PMCID: PMC5821286.

3. Apurba S Sastry, Sandhya Bhat. Essentials of Medical Microbiology. $3^{\text {th }}$ ed. New Delhi: Jaypeebrothers medical publishers; 2021.

4. Bitew, A., Molalign, T. \& Chanie, M. Species distribution and antibiotic susceptibility profile of bacterial uropathogens among patients complaining urinary tract infections. BMC Infect Dis 17, 654 (2017). DOI: 10.1186/s12879-017-2743-8.

5. C. Manikandan and A. Amsath. Antibiotic susceptibility pattern of Escherichia coli isolated from urine samples in Pattukkottai, Tamil Nadu. Int.J.Curr.Microbiol.App.Sci. 2014; 3(10): 449-457.

6. Chatterjee N, Chatterjee C, Ghosh S, Mukhopadhyay M, Brahmachari R, Patar K. Pattern of urinary antibiograms in a tertiary care hospital of eastern India. Journal of the Association of Physicians of India. 2016;64(4):26-30.

7. R Ananthanarayan, C K Jayaram Paniker. Ananthanarayan and Paniker's textbook of Microbiology. $8^{\text {th }}$ ed. Hyderabad: Universities Press (India); 2009.

8. Indian council of medical research. SOP Bacteriology. Antimicrobial Resistance Surveillance and Research Network. $2{ }^{\text {nd }}$ Edition, 2019. Indian council of medical research, New Delhi, India.

9. Clinical and Laboratory standards Institute. Performance standards for antimicrobial testing; twenty eighth international supplement. CLSI document M100-S 28, Clinical and Laboratory Standards Institute, Wayne, PA.2018.

10. Prakash D, Saxena R S. Distribution and antimicrobial susceptibility pattern of bacterial pathogens causing urinary tract infection in urban community of meerut city, India. ISRN Microbiol. 2013 Oct 29;2013:749629. DOI:
10.1155/2013/749629. PMID: 24288649; PMCID: PMC3830820.

11. Kumar Gaurav, Tripathi Shailendra Mohan, Mishra Neeti, Siddique M. E. Prevalence of urinary tract infections in elderly patients attending a tertiary care hospital. International Journal of Contemporary Medical Research 2019;6(2):B13-B16.

DOI: 10.21276/ijcmr.2019.6.2.31.

12. Veeraraghavan B, Jesudason M R, Prakasah J A J, Anandan S, Sahni R D, Pragasam A K et al., Antimicrobial susceptibility profiles of gram-negative bacteria causing infections collected across India during 2014-2016: Study for monitoring antimicrobial resistance trend report. Indian J Med Microbiol. 2018 JanMar;36(1):32-36. doi: 10.4103/ijmm.IJMM_17_415.

13. Shailaja Sukumaran T. and Mohan Kumar A. Antimicrobial resistance among uropathogenic bacteria in rural Kerala, India. International Journal of Current Microbiology and Applied Sciences. 2017;6(9): 2287-96. doi: 10.20546/ijcmas.2017.609.280.

14. Angmo D, Nazir S, Fomda B A, Akhtar S, Benazir S, Bhat A, et al., Microbiological profile of urinary tract infections among patients attending a tertiary care Hospital. Journal of Medical Science and Clinical Research. 2018;6(6):298-303.

15. Yadav M, Pal R, Damrolien S, Khumanthem S D. Microbial spectrum of urinary tract infections and its antibiogram in a tertiary care hospital. Int J Res Med Sci 2017;5:2718-22.

16. Niranjan V, Malini A. Antimicrobial resistance pattern in Escherichia coli causing urinary tract infection among inpatients. Indian J Med Res 2014;139:945-8.

17. Khameneh Z R, Afshar A T. Antimicrobial susceptibility pattern of urinary tract pathogens. Saudi J Kidney Dis Transpl. 2009 Mar;20(2):251-3.

\section{How to cite this article:}

Pradha Velu, Ramya Rengaraj, Hemalatha Gurumurthy and Saleem Mohamed Ali. 2021. Evaluation of Antimicrobial Susceptibility Pattern of Pathogenic Bacteria Causing Urinary Tract Infection in A Teaching Hospital. Int.J.Curr.Microbiol.App.Sci. 10(09): 456-463. doi: https://doi.org/10.20546/ijcmas.2021.1009.053 